ISSN 1855-3966 (printed edn.), ISSN 1855-3974 (electronic edn.)

ARS MATHEMATICA CONTEMPORANEA 16 (2019) 349-358

https://doi.org/10.26493/1855-3974.1485.0b1

(Also available at http://amc-journal.eu)

\title{
Decomposition method related to saturated hyperball packings
}

\author{
Jenő Szirmai \\ Budapest University of Technology and Economics, Institute of Mathematics, \\ Department of Geometry, H-1521 Budapest, Hungary
}

Received 14 September 2017, accepted 28 November 2018, published online 22 January 2019

\begin{abstract}
In this paper we study the problem of hyperball (hypersphere) packings in 3-dimensional hyperbolic space. We introduce a new definition of the non-compact saturated ball packings with generalized balls (horoballs, hyperballs) and describe to each saturated hyperball packing, a new procedure to get a decomposition of 3-dimensional hyperbolic space $\mathbb{H}^{3}$ into truncated tetrahedra. Therefore, in order to get a density upper bound for hyperball packings, it is sufficient to determine the density upper bound of hyperball packings in truncated simplices.
\end{abstract}

Keywords: Hyperbolic geometry, hyperball packings, Dirichlet-Voronoi cell, packing density, Coxeter tilings.

Math. Subj. Class.: 52C17, 52C22, 52B15

\section{Introduction}

In $n$-dimensional hyperbolic space $\mathbb{H}^{n}(n \geq 2)$ there are 3 kinds of generalized "balls" (spheres): the usual balls (spheres), horoballs (horospheres) and hyperballs (hyperspheres).

The classical problems of ball packings and coverings with congruent generalized balls of hyperbolic spaces $\mathbb{H}^{n}$ are extensively discussed in the literature, however there are several essential open questions e.g.:

1. What are the optimal ball packing and covering configurations of usual spheres and what are their densities $(n \geq 3)$ (see [1, 5, 7, 12])?

2. The monotonicity of the density related to the Böröczky type ball configurations depending on the radius of the congruent balls $(n \geq 4)$ (see $[4,10])$.

E-mail address: szirmai@math.bme.hu (Jenő Szirmai) 
3. What are the optimal horoball packing and covering configurations and what are their densities allowing horoballs in different types $(n \geq 4)($ see $[3,8,9])$ ?

4. What are the optimal packing and covering arrangements using non-compact balls (horoballs and hyperballs) and what are their densities? These are the so-called hyphor packings and coverings (see [21]).

5. What are the optimal hyperball packing and covering configurations and what are their densities $(n \geq 3)$ ?

In this paper we study the $5^{\text {th }}$ question related to saturated, congruent hyperball packings in 3-dimensional hyperbolic space $\mathbb{H}^{3}$.

In the hyperbolic plane $\mathbb{H}^{2}$ the universal upper bound of the hypercycle packing density is $\frac{3}{\pi}$, proved by I. Vermes in [24] and the universal lower bound of the hypercycle covering density is $\frac{\sqrt{12}}{\pi}$ determined by I. Vermes in [25].

In [15] and [16] we studied the regular prism tilings (simply truncated Coxeter orthoscheme tilings) and the corresponding optimal hyperball packings in $\mathbb{H}^{n}(n=3,4)$ and we extended the method developed in the former paper [20] to 5-dimensional hyperbolic space. Moreover, their metric data and their densities have been determined. In paper [19] we studied the $n$-dimensional hyperbolic regular prism honeycombs and the corresponding coverings by congruent hyperballs and we determined their least dense covering densities. Furthermore, we formulated conjectures for the candidates of the least dense hyperball covering by congruent hyperballs in the 3 - and 5-dimensional hyperbolic space $(n \in \mathbb{N}, 3 \leq n \leq 5)$.

In [22] we discussed congruent and non-congruent hyperball (hypersphere) packings of the truncated regular tetrahedron tilings. These are derived from the Coxeter simplex tilings $\{p, 3,3\}(7 \leq p \in \mathbb{N})$ and $\{5,3,3,3,3\}$ in 3- and 5-dimensional hyperbolic space. We determined the densest hyperball packing arrangement and its density with congruent hyperballs in $\mathbb{H}^{5}$ and determined the smallest density upper bounds of non-congruent hyperball packings generated by the above tilings in $\mathbb{H}^{n}(n=3,5)$.

In [21] we deal with packings derived by horo- and hyperballs (briefly hyp-hor packings) in $n$-dimensional hyperbolic spaces $\mathbb{H}^{n}(n=2,3)$ which form a new class of the classical packing problems. We constructed in the 2- and 3-dimensional hyperbolic spaces hyp-hor packings that are generated by complete Coxeter tilings of degree 1 i.e. the fundamental domains of these tilings are simple frustum orthoschemes and we determined their densest packing configurations and their densities. We proved using also numerical approximation methods that in the hyperbolic plane $(n=2)$ the density of the above hyp-hor packings arbitrarily approximate the universal upper bound of the hypercycle or horocycle packing density $\frac{3}{\pi}$ and in $\mathbb{H}^{3}$ the optimal configuration belongs to the $\{7,3,6\}$ Coxeter tiling with density $\approx 0.83267$. Furthermore, we analyzed the hyp-hor packings in truncated orthoschemes $\{p, 3,6\}(6<p<7, p \in \mathbb{R})$ whose density function is attained its maximum for a parameter which lies in the interval $[6.05,6.06]$ and the densities for parameters lying in this interval are larger that $\approx 0.85397$. That means that these locally optimal hyphor configurations provide larger densities that the Böröczky-Florian density upper bound $(\approx 0.85328)$ for ball and horoball packings but these hyp-hor packing configurations can not be extended to the entirety of hyperbolic space $\mathbb{H}^{3}$.

In [23] we studied a large class of hyperball packings in $\mathbb{H}^{3}$ that can be derived from truncated tetrahedron tilings. In order to get a density upper bound for the above hyperball packings, it is sufficient to determine this density upper bound locally, e.g. in truncated 
tetrahedra. Thus, we proved that if the truncated tetrahedron is regular, then the density of the densest packing is $\approx 0.86338$. This is larger than the Böröczky-Florian density upper bound for balls and horoballs but our locally optimal hyperball packing configuration cannot be extended to the entirety of $\mathbb{H}^{3}$. However, we described a hyperball packing construction, by the regular truncated tetrahedron tiling under the extended Coxeter group $\{3,3,7\}$ with maximal density $\approx 0.82251$.

Recently, (to the best of author's knowledge) the candidates for the densest hyperball (hypersphere) packings in the 3,4 and 5-dimensional hyperbolic space $\mathbb{H}^{n}$ are derived by the regular prism tilings that have been published in papers [15, 16] and [20].

In this paper we study hyperball (hypersphere) packings in 3-dimensional hyperbolic space. We develope a decomposition algorithm that for each saturated hyperball packing provides a decomposition of $\mathbb{H}^{3}$ into truncated tetrahedra. Therefore, in order to get a density upper bound for hyperball packings, it is sufficient to determine the density upper bound of hyperball packings in truncated simplices.

\section{Projective model and saturated hyperball packings in $\mathbb{H}^{3}$}

We use for $\mathbb{H}^{3}$ (and analogously for $\mathbb{H}^{n}, n \geq 3$ ) the projective model in the Lorentz space $\mathbb{E}^{1,3}$ that denotes the real vector space $\mathbf{V}^{4}$ equipped with the bilinear form of signature $(1,3)$,

$$
\langle\mathbf{x}, \mathbf{y}\rangle=-x^{0} y^{0}+x^{1} y^{1}+x^{2} y^{2}+x^{3} y^{3},
$$

where the non-zero vectors

$$
\mathbf{x}=\left(x^{0}, x^{1}, x^{2}, x^{3}\right) \in \mathbf{V}^{4} \quad \text { and } \quad \mathbf{y}=\left(y^{0}, y^{1}, y^{2}, y^{3}\right) \in \mathbf{V}^{4},
$$

are determined up to real factors, for representing points of $\mathcal{P}^{n}(\mathbb{R})$. Then $\mathbb{H}^{3}$ can be interpreted as the interior of the quadric $Q=\left\{(\mathbf{x}) \in \mathcal{P}^{3} \mid\langle\mathbf{x}, \mathbf{x}\rangle=0\right\}=: \partial \mathbb{H}^{3}$ in the real projective space $\mathcal{P}^{3}\left(\mathbf{V}^{4}, \boldsymbol{V}_{4}\right)$ (here $\boldsymbol{V}_{4}$ is the dual space of $\mathbf{V}^{4}$ ). Namely, for an interior point $\mathbf{y}$ holds $\langle\mathbf{y}, \mathbf{y}\rangle<0$.

Points of the boundary $\partial \mathbb{H}^{3}$ in $\mathcal{P}^{3}$ are called points at infinity, or at the absolute of $\mathbb{H}^{3}$. Points lying outside $\partial \mathbb{H}^{3}$ are said to be outer points of $\mathbb{H}^{3}$ relative to $Q$. Let $(\mathbf{x}) \in \mathcal{P}^{3}$, a point $(\mathbf{y}) \in \mathcal{P}^{3}$ is said to be conjugate to $(\mathbf{x})$ relative to $Q$ if $\langle\mathbf{x}, \mathbf{y}\rangle=0$ holds. The set of all points which are conjugate to $(\mathbf{x})$ form a projective (polar) hyperplane $\operatorname{pol}(\mathbf{x}):=\{(\mathbf{y}) \in$ $\left.\mathcal{P}^{3} \mid\langle\mathbf{x}, \mathbf{y}\rangle=0\right\}$. Thus, the quadric $Q$ induces a bijection (linear polarity $\mathbf{V}^{4} \rightarrow \boldsymbol{V}_{4}$ ) from the points of $\mathcal{P}^{3}$ onto their polar hyperplanes.

Point $X(\mathbf{x})$ and hyperplane $\alpha(\boldsymbol{a})$ are incident if $\mathbf{x} \boldsymbol{a}=0\left(\mathbf{x} \in \mathbf{V}^{4} \backslash\{\mathbf{0}\}, \boldsymbol{a} \in \boldsymbol{V}_{4} \backslash\{\mathbf{0}\}\right)$.

The hypersphere (or equidistance surface) is a quadratic surface at a constant distance from a plane (base plane) in both halfspaces. The infinite body of the hypersphere, containing the base plane, is called hyperball.

The half hyperball with distance $h$ to a base plane $\beta$ is denoted by $\mathcal{H}_{+}^{h}$. The volume of a bounded hyperball piece $\mathcal{H}_{+}^{h}(\mathcal{A})$, delimited by a 2-polygon $\mathcal{A} \subset \beta$, and its prism orthogonal to $\beta$, can be determined by the classical formula (2.1) of J. Bolyai [2].

$$
\operatorname{Vol}\left(\mathcal{H}_{+}^{h}(\mathcal{A})\right)=\frac{1}{4} \operatorname{Area}(\mathcal{A})\left[k \sinh \frac{2 h}{k}+2 h\right]
$$

The constant $k=\sqrt{\frac{-1}{K}}$ is the natural length unit in $\mathbb{H}^{3}$, where $K$ denotes the constant negative sectional curvature. In the following we may assume that $k=1$. 
Let $\mathcal{B}^{h}$ be a hyperball packing in $\mathbb{H}^{3}$ with congruent hyperballs of height $h$.

The notion of saturated packing follows from that fact that the density of any packing can be improved by adding further packing elements as long as there is sufficient room to do so. However, we usually apply this notion for packings with congruent elements. Now,

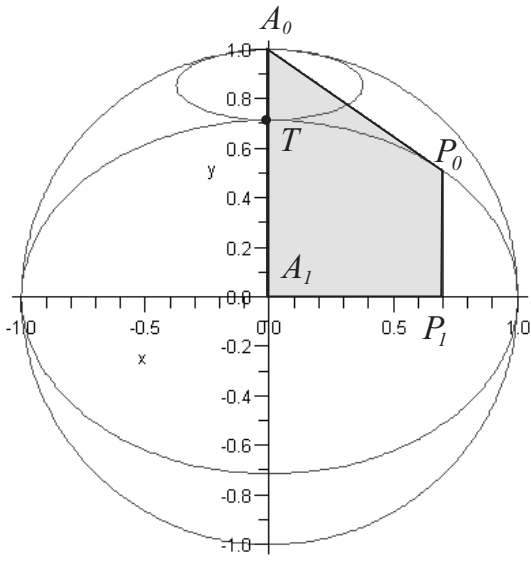

(a)

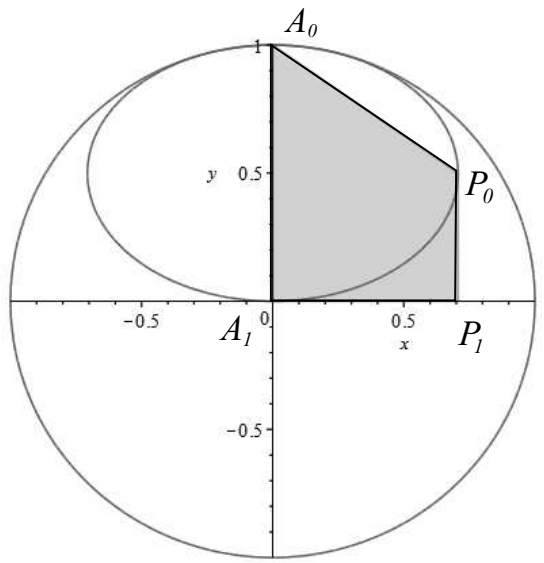

(b)

Figure 1: (a) Saturated hyp-hor packing, at present $a=0.7$. (b) Saturated horocycle packing with parameter $a=\frac{1}{\sqrt{2}}$.

we modify the classical definition of saturated packing for non-compact ball packings with generalized balls (horoballs, hyperballs) in $n$-dimensional hyperbolic space $\mathbb{H}^{n}(n \geq 2$ integer parameter):

Definition 2.1. A ball packing with non-compact balls (horoballs or/and hyperballs) in $\mathbb{H}^{n}$ is saturated if no new non-compact ball can be added to it.

We illustrate the meaning of the above definition by 2-dimensional Coxeter tilings given by the Coxeter symbol [ $\infty$ ] (see Figure 1), which are denoted by $\mathcal{T}_{a}$. The fundamental domain of $\mathcal{T}_{a}$ is a Lambert quadrilateral $A_{0} A_{1} P_{0} P_{1}$ (see [21]) that is denoted by $\mathcal{F}_{a}$. It is derived by the truncation of the orthoscheme $A_{0} A_{1} A_{2}$ by the polar line $\pi$ of the outer vertex $A_{2}$. The other initial principal vertex $A_{0}$ of the orthoscheme is lying on the absolute quadric of the Beltrami-Cayley-Klein model.

The images of $\mathcal{F}_{a}$ under reflections on its sides fill the hyperbolic plane $\mathbb{H}^{2}$ without overlap. The tilings $\mathcal{T}_{a}$ contain a free parameter $0<a<1, a \in \mathbb{R}$. The polar straight line of $A_{2}$ is $\pi$ and $\pi \cap A_{0} A_{2}=P_{0}, \pi \cap A_{1} A_{2}=P_{1}$. If we fix the parameter $a$ then a optimal hypercycle tiling can be derived from the mentioned Coxeter tiling (see Figure 1(a)) but here there are sufficient rooms to add horocycles with centre $A_{0}$ and with centres at the images of $A_{0}$. This saturated hyp-hor packing (packing with horo- and hyperballs) is illustrated in Figure 1(a). The Figure 1(b) shows a saturated horocycle packing belonging to the same Coxeter tiling.

To obtain hyperball (hypersphere) packing bounds it obviously suffices to study saturated hyperball packings (using the above definition) and in what follows we assume that all packings are saturated unless otherwise stated. 


\section{Decomposition into truncated tetrahedra}

We take the set of hyperballs $\left\{\mathcal{H}_{i}^{h}\right\}$ of a saturated hyperball packing $\mathcal{B}^{h}$ (see Definition 2.1). Their base planes are denoted by $\beta_{i}$. Thus, in a saturated hyperball packing the distance between two ultraparallel base planes $d\left(\beta_{i}, \beta_{j}\right)$ is at least $2 h$ (where for the natural indices holds $i<j$ and $d$ is the hyperbolic distance function).

In this section we describe a procedure to get a decomposition of 3-dimensional hyperbolic space $\mathbb{H}^{3}$ into truncated tetrahedra corresponding to a given saturated hyperball packing.

Step 1. The notion of the radical plane (or power plane) of two Euclidean spheres can be extended to the hyperspheres. The radical plane (or power plane) of two non-intersecting hyperspheres is the locus of points at which tangents drawn to both hyperspheres have the same length (so these points have equal power with respect to the two non-intersecting hyperspheres). If the two non-intersecting hyperspheres are congruent also in Euclidean sense in the model then their radical plane coincides with their "Euclidean symmetry plane" and any two congruent hypersphere can be transformed into such an hypersphere arrangement.

Using the radical planes of the hyperballs $\mathcal{H}_{i}^{h}$, similarly to the Euclidean space, can be constructed the unique Dirichlet-Voronoi (in short D-V) decomposition of $\mathbb{H}^{3}$ to the given hyperball packing $\mathcal{B}^{h}$. Now, the D-V cells are infinite hyperbolic polyhedra containing the corresponding hyperball, and its vertices are proper points of $\mathbb{H}^{3}$. We note here (it is easy to see), that a vertex of any D-V cell cannot be outer or boundary point of $\mathbb{H}^{3}$ relative to $Q$, because the hyperball packing $\mathcal{B}^{h}$ is saturated by the Definition 2.1 .

Step 2. We consider an arbitrary proper vertex $P \in \mathbb{H}^{3}$ of the above D-V decomposition and the hyperballs $\mathcal{H}_{i}^{h}(P)$ whose D-V cells meet at $P$. The base planes of the hyperballs $\mathcal{H}_{i}^{h}(P)$ are denoted by $\beta_{i}(P)$, and these planes determine a non-compact polyhedron $\mathcal{D}^{i}(P)$ with the intersection of their halfspaces containing the vertex $P$. Moreover, denote $A_{1}, A_{2}, A_{3}, \ldots$ the outer vertices of $\mathcal{D}^{i}(P)$ and cut off $\mathcal{D}^{i}(P)$ with the polar planes $\alpha_{j}(P)$ of its outer vertices $A_{j}$. Thus, we obtain a convex compact polyhedron $\mathcal{D}(P)$. This is bounded by the base planes $\beta_{i}(P)$ and "polar planes" $\alpha_{j}(P)$. Applying this procedure for all vertices of the above Dirichlet-Voronoi decomposition, we obtain an other decomposition of $\mathbb{H}^{3}$ into convex polyhedra.

Step 3. We consider $\mathcal{D}(P)$ as a tile of the above decomposition. The planes from the finite set of base planes $\left\{\beta_{i}(P)\right\}$ are called adjacent if there is a vertex $A_{s}$ of $\mathcal{D}^{i}(P)$ that lies on each of the above plane. We consider non-adjacent planes $\beta_{k_{1}}(P), \beta_{k_{2}}(P), \beta_{k_{3}}(P), \ldots$, $\beta_{k_{m}}(P) \in\left\{\beta_{i}(P)\right\}\left(k_{l} \in \mathbb{N}^{+}, l=1,2,3, \ldots, m\right)$ that have an outer point of intersection denoted by $A_{k_{1} \cdots k_{m}}$. Let $N_{\mathcal{D}(P)} \in \mathbb{N}$ denote the finite number of the outer points $A_{k_{1} \cdots k_{m}}$ related to $\mathcal{D}(P)$. It is clear, that its minimum is 0 if $\mathcal{D}^{i}(P)$ is tetrahedron. The polar plane $\alpha_{k_{1} \cdots k_{m}}$ of $A_{k_{1} \cdots k_{m}}$ is orthogonal to planes $\beta_{k_{1}}(P), \beta_{k_{2}}(P), \ldots, \beta_{k_{m}}(P)$ (thus, it contains their poles $\left.B_{k_{1}}, B_{k_{2}}, \ldots, B_{k_{m}}\right)$ and divides $\mathcal{D}(P)$ into two convex polyhedra $\mathcal{D}_{1}(P)$ and $\mathcal{D}_{2}(P)$.

Step 4. If $N_{\mathcal{D}_{1}(P)} \neq 0$ and $N_{\mathcal{D}_{2}(P)} \neq 0$ then $N_{\mathcal{D}_{1}(P)}<N_{\mathcal{D}(P)}$ and $N_{\mathcal{D}_{2}(P)}<N_{\mathcal{D}(P)}$ then we apply the Step 3 for polyhedra $\mathcal{D}_{i}(P), i \in\{1,2\}$.

Step 5. If $N_{\mathcal{D}_{i}(P)} \neq 0$ or $N_{\mathcal{D}_{j}(P)}=0$ ( $\left.i \neq j, i, j \in\{1,2\}\right)$ then we consider the polyhedron $\mathcal{D}_{i}(P)$ where $N_{\mathcal{D}_{i}(P)}=N_{\mathcal{D}(P)}-1$ because the vertex $A_{k_{1} \ldots k_{m}}$ is left out and apply the Step 3. 
Step 6. If $N_{\mathcal{D}_{1}(P)}=0$ and $N_{\mathcal{D}_{2}(P)}=0$ then the procedure is over for $\mathcal{D}(P)$. We continue the procedure with the next cell.

Step 7. It is clear, that the above plane $\alpha_{k_{1} \cdots k_{m}}$ intersects every hyperball $\mathcal{H}_{j}^{h}(P)$ $\left(j=k_{1}, \ldots, k_{m}\right)$.

Lemma 3.1. The plane $\alpha_{k_{1} \cdots k_{m}}$ of $A_{k_{1} \cdots k_{m}}$ does not intersect the hyperballs $\mathcal{H}_{s}^{h}(P)$ where $A_{k_{1} \cdots k_{m}} \notin \beta_{s}(P)$.

Proof. Let $\mathcal{H}_{s}^{h}(P)\left(A_{k_{1} \cdots k_{m}} \notin \beta_{s}(P)\right)$ be an arbitrary hyperball corresponding to $\mathcal{D}(P)$ with base plane $\beta_{s}(P)$ whose pole is denoted by $B_{s}$. The common perpendicular $\sigma$ of the planes $\alpha_{k_{1} \cdots k_{m}}$ and $\beta_{s}(P)$ is the line through the point $A_{k_{1} \cdots k_{m}}$ and $B_{s}$. We take a plane $\kappa$ containing the above common perpendicular, and its intersections with $\alpha_{k_{1} \cdots k_{m}}$ and $\mathcal{H}_{s}^{h}(P)$ are denoted by $\phi$ and $\eta$. We obtain the plane arrangement illustrated in Figure 2 which coincides with the configuration that is investigated in [24]. There I. Vermes noticed that the straight line $\phi=\alpha_{k_{1} \cdots k_{m}} \cap \kappa$ does not intersect the hypercycle $\eta=\mathcal{H}_{s}^{h}(P) \cap \kappa$. The plane $\alpha_{k_{1} \cdots k_{m}}$ and the hyperball $\mathcal{H}_{s}^{h}(P)$ can be generated by rotation of $\phi$ and $\eta$ about the common perpendicular $\sigma$; therefore, they are disjoint.

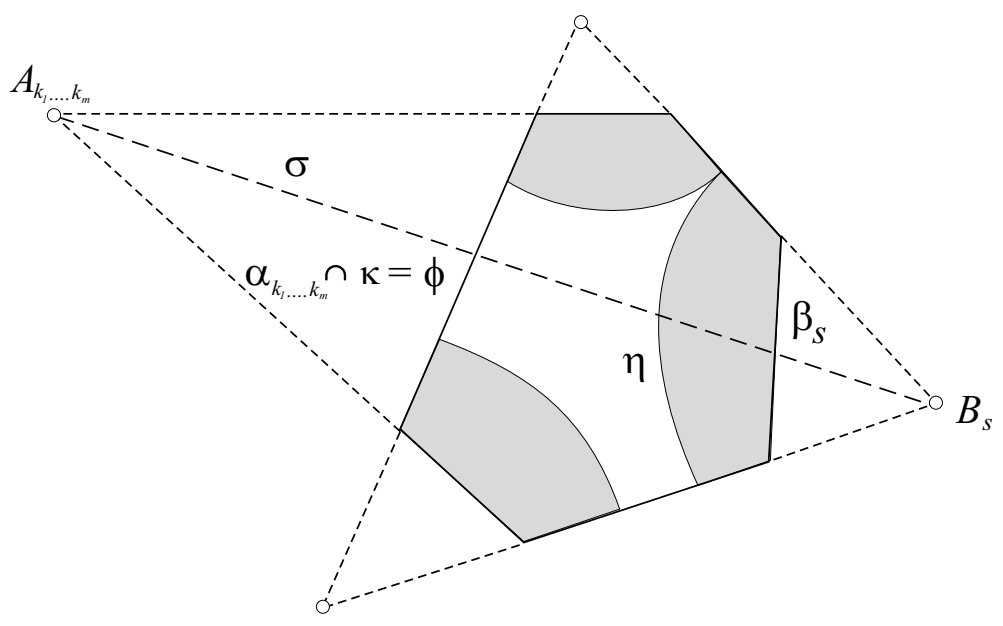

Figure 2: The plane $\kappa$ and its intersections with $\mathcal{D}(P)$ and $\mathcal{H}_{s}^{h}(P)$.

Step 8. We have seen in Steps 3, 4, 5 and 6 that the number of the outer vertices $A_{k_{1} \cdots k_{m}}$ of any polyhedron obtained after the cutting process is less than the original one, and we have proven in Step 7 that the original hyperballs form packings in the new polyhedra $\mathcal{D}_{1}(P)$ and $\mathcal{D}_{2}(P)$, as well. We continue the cutting procedure described in Step 3 for both polyhedra $\mathcal{D}_{1}(P)$ and $\mathcal{D}_{2}(P)$. If a derived polyhedron is a truncated tetrahedron then the cutting procedure does not give new polyhedra, thus the procedure will not be continued. Finally, after a finite number of cuttings we get a decomposition of $\mathcal{D}(P)$ into truncated tetrahedra, and in any truncated tetrahedron the corresponding congruent hyperballs from $\left\{\mathcal{H}_{i}^{h}\right\}$ form a packing. Moreover, we apply the above method for the further cells. 
Finally we get the following:

Theorem 3.2. The above described algorithm provides for each congruent saturated hyperball packing a decomposition of $\mathbb{H}^{3}$ into truncated tetrahedra.

The above procedure is illustrated for regular octahedron tilings derived by the regular prism tilings with Coxeter-Schläfli symbol $\{p, 3,4\}, 6<p \in \mathbb{N}$. These Coxeter tilings and the corresponding hyperball packings are investigated in [15]. In this situation the convex polyhedron $\mathcal{D}(P)$ is a truncated octahedron (see Figure 3 ) whose vertices $B_{i}$ ( $i=1,2,3,4,5,6)$ are outer points and the octahedron is cut off with their polar planes $\beta_{i}$. These planes are the base planes of the hyperballs $\mathcal{H}_{i}^{h}$. We can assume that the centre of the octahedron coincides with the centre of the model.

First, we choose three non-adjacent base planes $\beta_{2}, \beta_{3}, \beta_{4}$. Their common point, denoted by $A_{234}$ and its polar plane $\alpha_{234}$ are determined by points $B_{2}, B_{3}, B_{4}$ containing the centre $P$ as well. Then we consider the non-adjacent base planes $\beta_{2}, \beta_{4}, \beta_{5}$ and the polar plane $\alpha_{245}$ of their common point $A_{245}$. It is clear that the points $B_{2}, B_{4}, B_{5}$ lie in the plane $\alpha_{245}$ (see Figure 3).

By the above two "cuttings" we get the decomposition of $\mathcal{D}(P)$ into truncated simplices.

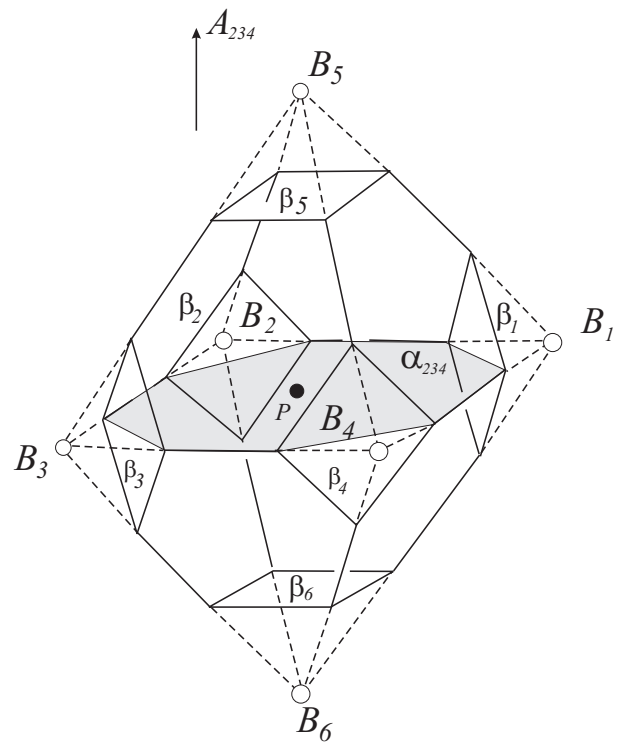

(a)

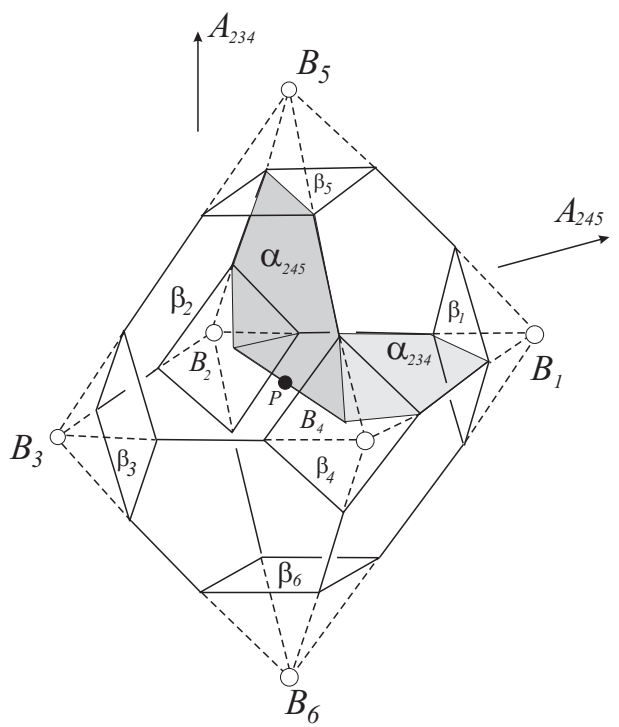

(b)

Figure 3: Truncated octahedron tiling derived from the regular prism tilings with CoxeterSchläfli symbol $\{p, 3,4\}$ and its decomposition into truncated tetrahedra.

\section{Remark 3.3.}

1. If we try to define the density of system of sets in hyperbolic space as we did in Euclidean space, i.e. by the limiting value of the density with respect to a sphere $C(r)$ of radius $r$ with a fixed centre $O$. But since for a fixed value of $h$ the volume 
of spherical shell $C(r+h)-C(r)$ is the same order of magnitude as the volume of $C(r)$, the argument used in Euclidean space to prove that the limiting value is independent of the choice of $O$ is does not work in hyperbolic space. Therefore the definition of packing density is crucial in hyperbolic spaces $\mathbb{H}^{n}$ as shown by K. Böröczky [3], for nice examples also see [6, 14]. The most widely accepted notion of packing density considers the local densities of balls with respect to their Dirichlet-Voronoi cells (cf. [3] and [7]), but in our cases these cells are infinite hyperbolic polyhedra. The other possibility: the packing density $\delta$ can be defined (see $[15,20,24,25]$ ) as the reciprocal of the ratio of the volume of a fundamental domain for the symmetry group of a tiling to the volume of the ball pieces contained in the fundamental domain $(\delta<1)$. Similarly is defined the covering density $\Delta>1$. In order to determine an upper bound for the density of congruent hyperball packings in $\overline{\mathbb{H}}^{n}$ we used an extended notion of such local density. Therefore, we had to construct a decomposition of $\mathbb{H}^{n}$ into compact cells to define local density to a given hyperball packing and these corresponding cells are (not absolutely congruent) truncated tetrahedra (see the above algorithm and [23]).

2. From the above section it follows that, to each saturated hyperball packing $\mathcal{B}^{h}$ of hyperballs $\mathcal{H}_{i}^{h}$ there is a decomposition of $\mathbb{H}^{3}$ into truncated tetrahedra. Therefore, in order to get a density upper bound for hyperball packings, it is sufficient to determine the density upper bound of hyperball packings in truncated simplices. We observed in [23] that some extremal properties of hyperball packings naturally belong to the regular truncated tetrahedron (or simplex, in general, see Lemma 3.2 and Lemma 3.3 in [23]). Therefore, we studied hyperball packings in regular truncated tetrahedra, and prove that if the truncated tetrahedron is regular, then the density of the densest packing is $\approx 0.86338$ (see Theorem 5.1 in [23]). However, these hyperball packing configurations are only locally optimal, and cannot be extended to the whole space $\mathbb{H}^{3}$. Moreover, we showed that the densest known hyperball packing, dually related to the regular prism tilings, introduced in [15], can be realized by a regular truncated tetrahedron tiling with density $\approx 0.82251$.

3. In [22] we discussed the problem of congruent and non-congruent hyperball (hypersphere) packings to each truncated regular tetrahedron tiling. These are derived from the Coxeter simplex tilings $\{p, 3,3\}$ and $\{5,3,3,3,3\}$ in the 3 - and 5 -dimensional hyperbolic space. We determined the densest hyperball packing arrangement and its density with congruent hyperballs in $\mathbb{H}^{5}(\approx 0.50514)$ and determined the smallest density upper bounds of non-congruent hyperball packings generated by the above tilings: in $\mathbb{H}^{3}(\approx 0.82251)$; in $\mathbb{H}^{5}(\approx 0.50514)$.

The question of finding the densest hyperball packings and horoball packings with horoballs of different types in the $n$-dimensional hyperbolic spaces $n \geq 3$ has not been settled yet either (see e.g. [8, 9, 13, 23]).

Optimal sphere packings in other homogeneous Thurston geometries represent another huge class of open mathematical problems. For these non-Euclidean geometries only very few results are known (e.g. [17, 18]). Detailed studies are the objective of ongoing research. The applications of the above projective method seem to be interesting in (non-Euclidean) crystallography as well, a topic of much current interest. 


\section{References}

[1] K. Bezdek, Sphere packings revisited, European J. Combin. 27 (2006), 864-883, doi:10.1016/ j.ejc.2005.05.001.

[2] J. Bolyai, Appendix: Scientiam spatii absolute veram exhibens, in: F. Bolyai (ed.), Tentamen juventutem studiosam in elementa matheseos purae, elementaris ac sublimioris, methodo intuitiva, evidentiaque huic propria, introducendi, Tomus primus, Maros Vásárhelyini: J. \& S. Kali, 1832.

[3] K. Böröczky, Packing of spheres in spaces of constant curvature, Acta Math. Acad. Sci. Hungar. 32 (1978), 243-261, doi:10.1007/bf01902361.

[4] K. Böröczky and A. Florian, Über die dichteste Kugelpackung im hyperbolischen Raum, Acta Math. Acad. Sci. Hungar. 15 (1964), 237-245, doi:10.1007/bf01897041.

[5] G. Fejes Tóth, G. Kuperberg and W. Kuperberg, Highly saturated packings and reduced coverings, Monatsh. Math. 125 (1998), 127-145, doi:10.1007/bf01332823.

[6] G. Fejes Tóth and W. Kuperberg, Packing and covering with convex sets, in: P. M. Gruber and J. M. Wills (eds.), Handbook of Convex Geometry, Volume B, North-Holland, Amsterdam, pp. 799-860, 1993, doi:10.1016/c2009-0-15706-9.

[7] R. Kellerhals, Ball packings in spaces of constant curvature and the simplicial density function, J. Reine Angew. Math. 494 (1998), 189-203, doi:10.1515/crll.1998.006.

[8] R. T. Kozma and J. Szirmai, Optimally dense packings for fully asymptotic Coxeter tilings by horoballs of different types, Monatsh. Math. 168 (2012), 27-47, doi:10.1007/ s00605-012-0393-x.

[9] R. T. Kozma and J. Szirmai, New lower bound for the optimal ball packing density in hyperbolic 4-space, Discrete Comput. Geom. 53 (2015), 182-198, doi:10.1007/s00454-014-9634-1.

[10] T. H. Marshall, Asymptotic volume formulae and hyperbolic ball packing, Ann. Acad. Sci. Fenn. Math. 24 (1999), 31-43, http://www.acadsci.fi/mathematica/Vol24/ marshall.html.

[11] E. Molnár, The projective interpretation of the eight 3-dimensional homogeneous geometries, Beiträge Algebra Geom. 38 (1997), 261-288, https : / / www • emis . de / journals / $\mathrm{BAG} / \mathrm{vol} .38 / \mathrm{no} .2 / 8 . \mathrm{html}$.

[12] E. Molnár and J. Szirmai, Top dense hyperbolic ball packings and coverings for complete Coxeter orthoscheme groups, Publ. Inst. Math. (Beograd) 103 (2018), 129-146, doi:10.2298/ pim1817129m.

[13] L. Németh, On the hyperbolic Pascal pyramid, Beiträge Algebra Geom. 57 (2016), 913-927, doi:10.1007/s13366-016-0293-7.

[14] C. Radin, The symmetry of optimally dense packings, in: A. Prékopa and E. Molnár (eds.), Non-Euclidean Geometries, Springer, New York, volume 581 of Mathematics and Its Applications (New York), 2006 pp. 197-207, doi:10.1007/0-387-29555-0_10, papers from the International Conference on Hyperbolic Geometry held in Budapest, July 6 - 12, 2002.

[15] J. Szirmai, The regular $p$-gonal prism tilings and their optimal hyperball packings in the hyperbolic 3-space, Acta Math. Hungar. 111 (2006), 65-76, doi:10.1007/s10474-006-0034-8.

[16] J. Szirmai, The regular prism tilings and their optimal hyperball packings in the hyperbolic $n$-space, Publ. Math. Debrecen 69 (2006), 195-207.

[17] J. Szirmai, A candidate for the densest packing with equal balls in Thurston geometries, Beiträge Algebra Geom. 55 (2014), 441-452, doi:10.1007/s13366-013-0158-2. 
[18] J. Szirmai, Simply transitive geodesic ball packings to $\mathbf{S}^{2} \times \mathbf{R}$ space groups generated by glide reflections, Ann. Mat. Pura Appl. 193 (2014), 1201-1211, doi:10.1007/s10231-013-0324-z.

[19] J. Szirmai, The least dense hyperball covering of regular prism tilings in hyperbolic $n$-space, Ann. Mat. Pura Appl. 195 (2016), 235-248, doi:10.1007/s10231-014-0460-0.

[20] J. Szirmai, The optimal hyperball packings related to the smallest compact arithmetic 5orbifolds, Kragujevac J. Math. 40 (2016), 260-270, doi:10.5937/kgjmath1602260s.

[21] J. Szirmai, Packings with horo- and hyperballs generated by simple frustum orthoschemes, Acta Math. Hungar. 152 (2017), 365-382, doi:10.1007/s10474-017-0728-0.

[22] J. Szirmai, Density upper bound for congruent and non-congruent hyperball packings generated by truncated regular simplex tilings, Rend. Circ. Mat. Palermo 67 (2018), 307-322, doi:10. 1007/s12215-017-0316-8.

[23] J. Szirmai, Hyperball packings in hyperbolic 3-space, Mat. Vesnik 70 (2018), 211-221, http: //www.vesnik.math.rs/vol/mv18303.pdf.

[24] I. Vermes, Ausfüllungen der hyperbolischen Ebene durch kongruente Hyperzykelbereiche, $\mathrm{Pe}$ riod. Math. Hungar. 10 (1979), 217-229, doi:10.1007/bf02020020.

[25] I. Vermes, Über reguläre Überdeckungen der Bolyai-Lobatschewskischen Ebene durch kongruente Hyperzykelbereiche, Period. Polytech. Mech. Engrg. 25 (1981), 249-261, https: //pp.bme.hu/me/article/view/5842. 